A further source of pleasure is provided by the admirable collection of illustrations, including a coloured frontispiece of the exquisite "Peacock in the Vase of Hermes" from a British Museum MS. of the "Splendor Solis". Alchemy relied very largely upon pictorial representation, so that its tenets cannot be properly appreciated without continued reference to such graphic aids; the "Mutus Liber" (included in Manget's "Bibliotheca Chemica Curiosa") claims indeed to set forth the whole of Hermetic philosophy in a series of unannotated pictures.

Count Michael Maier (1568-1622) pressed another of the arts into the service of alchemy, setting to music-presumably of his own compositionLatin epigrams on secrets of "Chymical Nature".
Prof. Read had the excellent idea of persuading a St. Andrews choir to learn and to sing some of these alchemical fugues, and since their rendering has been perpetuated in a gramophone record, anyone who wishes may hear it. In this part of his work he has had the assistance of Mr. F. H. Sawyer and Prof. H. J. Rose, whose modern chemical canon, "Hydrogenesis", written and composed in imitation of Maier's, shows 'a pretty wit': "Aëre cum crasso tenuis componitur aër; adde Iouis fulmen, fit sonus, unda manet. With a dense gas a thin gas is mixed; pass the electric spark, and pop! you have water." But it must have been Prof. Read who so thoughtfully added: " $2 \mathrm{H}_{2}+\mathrm{O}_{2}=2 \mathrm{H}_{2} \mathrm{O}$ "!

\section{E. J. HolmyaRD.}

\title{
Quantitative Organic Microanalysis
}

\section{Quantitative Organic Microanalysis of Fritz Pregl}

By Dr. Hubert Roth. Third English edition translated from the fourth revised and enlarged German edition by E. Beryl Daw. Pp. xvi +271. (London : J. and A. Churchill, Ltd., 1937.) 18s.

$\mathrm{T}^{\mathrm{H}}$ HE brilliant yet painstaking investigations of the late Prof. Pregl have placed quantitative organic microanalysis on such a sure footing that not only have such methods been widely adopted but also improvements and additions are constantly being introduced. Indeed, to-day, practically all the analytical procedures which the organic chemist is likely to require have been made available for him on the micro scale. This rapid development of the subject has made necessary a further edition of Pregl's well-known manual, which has now been brought up to date by Dr. Roth and translated into English by Miss Beryl Daw.

The book has been considerably enlarged and revised and, as hitherto, it opens with a section on the microchemical balance. To the matter in previous editions there have been added descriptions of a new Kuhlmann aperiodic balance, of two of the latest Bunge types, while a brief mention is made of ultra-microchemical balances.

The section dealing with elementary analysis has not been greatly modified. A method of estimating carbon in the wet way is given, while the micro-Kjeldahl determination of nitrogen has been revised and a detailed description given of Friedrich's incineration with hydriodic acid. Other new procedures inclure a wet decomposition of halogen derivatives; the determination of iodine as iodate; the estimation of sulphur in the presence of nitrogen, chlorine and bromine.

It is in the sections dealing respectively with the general groups and physical methods that the greatest alteration has been made, numerous new methods being included. These comprise the determination of active hydrogen by the Grignard reagent; the titration of amino acids; the volumetric determination of alkoxyl, alkylimino and S-alkyl groups; the estimation of C-methyl and of isopropylidine groups; catalytic microhydrogenation processes. New physical methods which are given include the microscopic determination of melting points; the adaptation of Schleiermacher's principle to 'micro-boiling points'; the determination of molecular weights by Barger's method of isothermal distillation; the photoelectric measurement of absorption spectra; the determinations of molecular refraction and specific rotation.

In the descriptions of the various procedures, there is given first a brief historical survey of the estimation, after which the apparatus and materials used are discussed. Then follows a very lucid and detailed summary of the course of the determination, while, finally, an actual sample analysis is given.

The translator has performed her task quite creditably, although a number of small errors have been allowed to creep into the text. Fortunately, these are relatively unimportant, and do not detract from the great value of the book both to the organic chemist and to the analyst.

G. R. D. 